\title{
The Change of Laboratory Tests Could Be Predictive Factors for Infection after McKeown Esophagogastrectomy
}

\author{
Chongxiang Chen, ${ }^{1,2}$ Tianmeng Wen $\mathbb{D}^{3},{ }^{3}$ and Qingyu Zhao $\mathbb{D}^{1}$ \\ ${ }^{1}$ Department of Intensive Care Unit, Sun Yat-Sen University Cancer Center, State Key Laboratory of Oncology in South China, \\ Collaborative Innovation Center for Cancer Medicine, Guangzhou, 510060, China \\ ${ }^{2}$ Guangzhou Institute of Respiratory Diseases, State Key Laboratory of Respiratory Disease, \\ The First Affiliated Hospital of Guangzhou Medical University, Guangzhou, 510120, China \\ ${ }^{3}$ School of Public Health, Sun Yat-Sen University, Guangzhou, Guangdong Province, China \\ Correspondence should be addressed to Qingyu Zhao; zhaoqy163@163.com
}

Received 20 April 2019; Accepted 18 July 2019; Published 22 October 2019

Guest Editor: Yatao Liu

Copyright (c) 2019 Chongxiang Chen et al. This is an open access article distributed under the Creative Commons Attribution License, which permits unrestricted use, distribution, and reproduction in any medium, provided the original work is properly cited.

\begin{abstract}
Aim. To analyze whether the change of laboratory tests (postoperative day 1 (POD1) minus pre-operation) could be predictive factors for postoperative infection in patients who have undergone McKeown esophagogastrectomy. Methods. We retrospectively investigated the clinical data of 358 patients who have undergone McKeown esophagogastrectomy, and divided them into infection and noninfection groups. SPSS 22.0 software was performed for data analysis. Results. In the two groups, smoking status (66.7\% vs. $42.3 \% ; P=0.014)$, male gender ( $86.1 \%$ vs. $72.0 \% ; P<0.001)$, hoarseness $(23.6 \%$ vs. $8.7 \% ; P<0.001)$, poor coughing ability (51.4\% vs. $9.1 \% ; P<0.001)$, the change of WBC count $\left(5.59 \pm 4.75 \times 10^{9} / \mathrm{L}\right.$ vs. $\left.4.51 \pm 4.11 \times 10^{9} / \mathrm{L} ; P=0.05\right)$, the change of glucose $(6.03 \pm 3.97 \mathrm{~g} / \mathrm{L}$ vs. $3.78 \pm 3.18 \mathrm{~g} / \mathrm{L})$, the change of ALB $(-12.83 \pm 3.45 \mathrm{~g} / \mathrm{L}$ vs. $-10.69 \pm 3.86 \mathrm{~g} / \mathrm{L})$, the change of CRE $(0.17 \pm 19.94 \mathrm{umol} / \mathrm{L}$ vs. $-4.02 \pm 15.40 \mathrm{umol} / \mathrm{L}, P=0.047$ ) were significantly different. These factors were assessed using logistic regression analysis, and factors with $P \leq 0.05$ in the univariate analysis were entered into multivariate analysis based on the forward stepwise (conditional) method. Poor coughing ability (odds ratio [OR], 11.034, 95\% confidence interval [CI], 5.358-22.724), smoking status (OR, 4.218; 95\% CI, 2.110-8.431), the change of WBC count (OR, 1.079; 95\% CI, 1.000-1.164), the change of serum ALB level (OR, 0.849; 95\% CI, 0.772-0.935), and the change of blood glucose levels (OR, 1.237; 95\% CI, 1.117-1.371) were determined as independent risk factors for postoperative infection. We established a scoring system based on these 5 factors, and the area under the curve for this predictive model was 0.843 (range, $0.793-0.894$ ); the sensitivity, specificity, and cut-off score were $70.8 \%, 85.3 \%$, and 2.500 , respectively. Conclusion. Poor coughing ability, smoking habit, the high change of WBC and blood glucose levels, and low change of serum ALB levels can be used to predict the occurrence of postoperative infections among patients who have undergone McKeown esophagogastrectomy.
\end{abstract}

\section{Introduction}

Up to now, Esophageal cancer is the sixth-most common cause of cancer-related death all around the world, and in developing countries, it is the fifth most frequent cause of deaths [1]. Furthermore, the incidence and mortality of patients with esophageal cancer in China were the highest globally in 2009 [2]. Surgery remains the standard treatment for resectable esophageal cancer. However, esophagogastrectomy is a complex procedure, with morbidity and mortality rates of $23 \%-$ $50 \%$ and $2 \%-8 \%$, respectively $[3,4]$.
Patients undergoing McKeown esophagogastrectomy are exposed to a higher risk of infection compared with those receiving other types of surgery. Moreover, patients with esophageal cancer are at a greater risk of antimicrobial exposure due to their impaired immunological functions and are also at an increased risk of infection with multidrug-resistant bacteria.

In this study, we assumed that the change of laboratory tests (laboratory tests within $24 \mathrm{~h}$ after surgery minus pre-operation) will be associated with the infections following McKeown esophagogastrectomy, and developed recommendations for clinicians treating patients with these risk factors. 


\section{Methods}

2.1. Data Collection. We collected clinical data from 358 esophageal cancer patients (including 268 male and 90 female patients) who were admitted for McKeown esophagogastrectomy (right thoracotomy followed by laparotomy and cervical anastomosis) between July 2014 and October 2016 at Sun Yat-Sen University Cancer Center (SYSUCC). The RDD number for this study is RDDA2019001127. The average age of the patients was $60.55 \pm 7.87$ years. We divided the patients into the infection and noninfection groups according to the occurrence of postoperative infections, and then retrospectively assessed the baseline characteristics, clinical disease features, perioperative features, preoperative, and postoperative laboratory test results (including white blood cell [WBC], neutrophil, hemoglobin $[\mathrm{HB}]$, aspartate aminotransferase [AST], alanine aminotransferase [ALT], serum albumin [ALB], blood urea nitrogen [BUN], creatinine [CRE], blood glucose, C-reactive protein [CRP], and lactic acid levels) between the groups. All the postoperative day 1 (POD1) indicators were analyzed within $24 \mathrm{~h}$ after surgery. And we used POD1 indicators minus preoperative ones to calculate the change of laboratory tests.

2.2. Inclusion and Exclusion Criteria. The inclusion criteria were as follows: patients aged $>18$ years with esophageal cancer who underwent McKeown esophagogastrectomy and developed an infection during hospitalization. And those with infection prior to hospital admission were excluded from the study.

2.3. Statistical Analysis. Categorical variables were expressed as number and percentage, and continuous variables were expressed as mean \pm standard deviation. Student's $t$-test was used to examine continuous variables, and the Chi-squared test or Fisher's exact test was used to assess categorical variables. Multi-variate analysis was performed to determine the predictors of postoperative infection, and the forward stepwise (conditional) method was used to identify factors to enter into the multivariate regression model. Receiver operating characteristic (ROC) curves were constructed to estimate the sensitivity, specificity, and the area under the curve (AUC) for various cutoff points of the relevant indicators. Statistical significance was set at $P \leq 0.05$, and all statistical analyses were computed using SPSS Version 22.0.

\section{Results}

3.1. Differences in the Baseline Characteristics. Table 1 describes the characteristics of the 72 patients $(20.1 \%)$ with postoperative infection, from among the 358 patients who had undergone McKeown esophagogastrectomy in the present study. We compared the patients' baseline characteristics and clinical disease features between groups, and identified significant differences in smoking habits and gender between the two groups. The smoking habit frequency $(66.7 \% \mathrm{vs}$. $42.3 \% ; P<0.001)$ and proportion of males $(86.1 \%$ vs. $72.0 \%$; $P=0.014)$ were greater in the infection group than in the noninfection group (Table 1 and Figure 1).
Graph of percent of the baseline characteristics

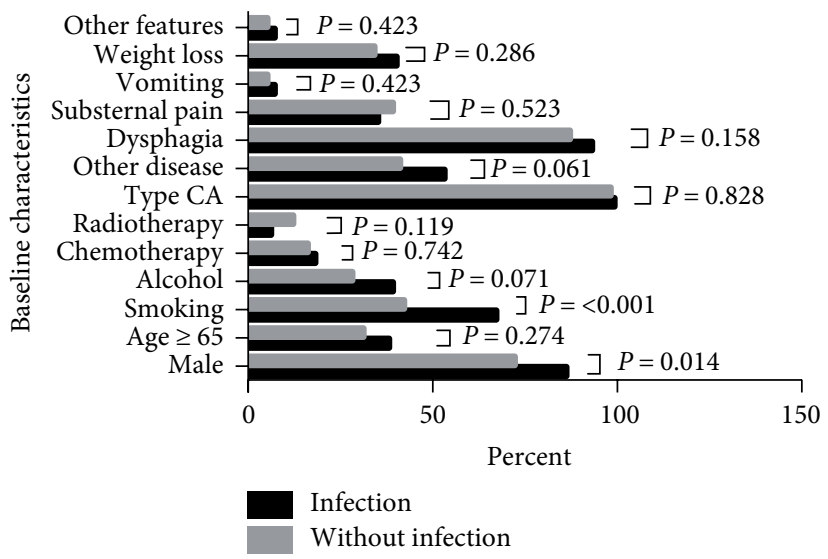

FIgUre 1: Baseline characteristics and clinical disease features between the infection group and noninfection group.

Graph of percent of perioperative features

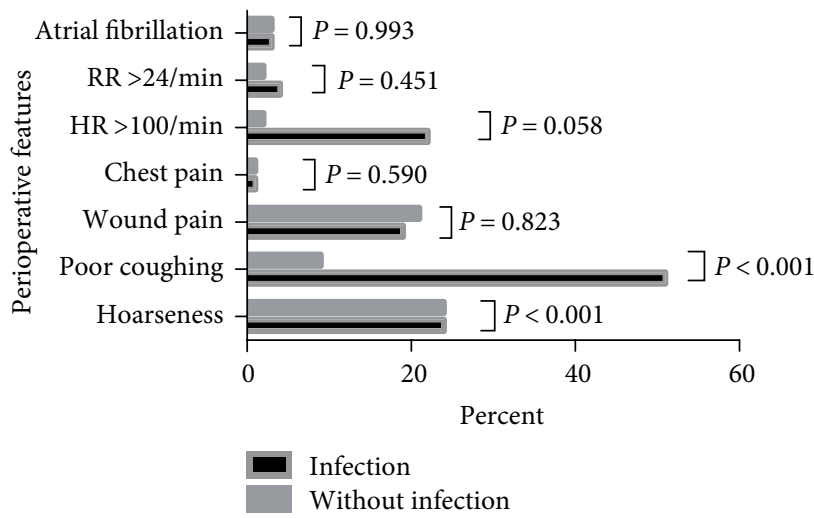

Figure 2: Difference in perioperative features among patients who underwent McKeon esophagogastrectomy.

3.2. Differences in the Perioperative Clinical Features. In the present study, the factors of hoarseness $(23.6 \%$ vs. $8.7 \%$; $P=0.001)$ and poor coughing ability (51.4\% vs. $9.1 \%$; $P<0.001)$ were significantly different between the groups; both were more frequent in the infection group. However, other perioperative clinical features, including wound pain, increased heart rate and respiratory rate, chest pain/chest distress, and atrial fibrillation, did not exhibit a significant difference (Table 2 and Figure 2).

3.3. Differences in Change of Laboratory Test Results. The results of the change of laboratory tests (POD1 minus pre-operation) were compared between the groups. The change of WBC count $\left(5.59 \pm 4.75 \times 10^{9} / \mathrm{L}\right.$ vs. $\left.4.47 \pm 4.14 \times 10^{9} / \mathrm{L} ; P=0.048\right)$, neutropil count $\left(7.00 \pm 4.63 \times 10^{9} / \mathrm{L}\right.$ vs. $\left.5.83 \pm 3.69 \times 10^{9} / \mathrm{L} ; P=0.023\right)$, glucose $(6.03 \pm 3.97 \mathrm{~g} / \mathrm{L}$ vs. $3.78 \pm 3.18 \mathrm{~g} / \mathrm{L} ; P<0.001), \mathrm{ALB}$ $(-12.83 \pm 3.45 \mathrm{~g} / \mathrm{L}$ vs. $-10.69 \pm 3.86 \mathrm{~g} / \mathrm{L} ; \quad P<0.001), \quad \mathrm{CRE}$ $(0.17 \pm 19.94 \mathrm{umol} / \mathrm{L}$ vs. $-4.02 \pm 15.40 \mathrm{umol} / \mathrm{L}, P=0.047)$ were greater in the infection group than in the noninfection group. None of the other change of laboratory test results showed significant differences (Table 3 and Figure 3). 
TABLE 1: Baseline characteristics and clinical disease features between the infection group and noninfection group.

\begin{tabular}{|c|c|c|c|c|}
\hline Outcome & Infection group (\%) & Noninfection group (\%) & $\chi^{2}$ & $P$ value \\
\hline Sum & 72 & 286 & & \\
\hline Gender & & & 6.062 & $0.014^{*}$ \\
\hline Male & $62(86.1)$ & $206(72.0)$ & & \\
\hline Female & $10(13.9)$ & $80(28.0)$ & & \\
\hline Age & & & 1.195 & 0.274 \\
\hline$\geq 65$ & $27(37.5)$ & $88(30.8)$ & & \\
\hline$<65$ & $45(62.5)$ & $198(69.2)$ & & \\
\hline Smoking habit & & & 13.695 & $<0.001^{*}$ \\
\hline Yes & $48(66.7)$ & $121(42.3)$ & & \\
\hline No & $24(33.3)$ & $165(57.7)$ & & \\
\hline Alcohol consumption & & & 3.254 & 0.071 \\
\hline Yes & $28(38.9)$ & $80(28.0)$ & & \\
\hline No & $44(61.1)$ & $206(72.0)$ & & \\
\hline Chemotherapy & & & 0.108 & 0.742 \\
\hline Yes & $13(18.1)$ & $47(16.4)$ & & \\
\hline No & $59(81.9)$ & $239(83.6)$ & & \\
\hline Radiotherapy & & & 2.431 & 0.119 \\
\hline Yes & $4(5.6)$ & $34(11.9)$ & & \\
\hline No & $68(94.4)$ & $252(88.1)$ & & \\
\hline Type of cancer & & & 0.047 & 0.828 \\
\hline Squamous & $71(98.6)$ & $281(98.3)$ & & \\
\hline Others & $1(1.4)$ & $5(1.7)$ & & \\
\hline Other chronic disease & & & 3.503 & 0.061 \\
\hline Yes & $38(52.8)$ & $116(40.6)$ & & \\
\hline No & $34(48.2)$ & $170(59.4)$ & & \\
\hline Dysphagia & & & 1.995 & 0.158 \\
\hline Yes & $67(93.1)$ & $249(87.1)$ & & \\
\hline No & $5(6.9)$ & $37(12.9)$ & & \\
\hline Substernal pain & & & 0.408 & 0.523 \\
\hline Yes & $25(34.7)$ & $111(38.8)$ & & \\
\hline No & $47(65.3)$ & $175(61.2)$ & & \\
\hline Acid regurgitation/vomiting & & & 0.641 & 0.423 \\
\hline Yes & $5(6.9)$ & $13(4.5)$ & & \\
\hline No & $67(93.1)$ & $273(95.5)$ & & \\
\hline Weight loss & & & 1.140 & 0.286 \\
\hline Yes & $29(40.3)$ & $96(33.6)$ & & \\
\hline No & $43(59.7)$ & $190(66.4)$ & & \\
\hline Other clinical features & & & 0.641 & 0.423 \\
\hline Yes & $5(6.9)$ & $13(4.5)$ & & \\
\hline No & $67(93.1)$ & $273(95.5)$ & & \\
\hline
\end{tabular}

*Statistically significant at $P \leq 0.05$.

3.4. Multivariate Analysis. Factors that were significant in the univariate analysis $(P<0.05)$ were included in the multivariate analysis. Accordingly, we assessed 5 factors, including poor coughing ability (odds ratio [OR], 11.034; 95\% confidence interval CI, 5.358-22.724), smoking status (OR, 4.218; 95\% CI, 2.110-8.431), the change of WBC count (OR, 1.079; 95\% CI, 1.000-1.164), ALB level (OR, 0.849; 95\% CI, 0.772-0.935), blood glucose level (OR, 1.237; 95\% CI, 1.117-1.371), using multivariate regression; male gender and the other laboratory test results were not included (Table 4).

\subsection{Development of a Scoring System to Predict Postoperative} Infections. The AUC and cut-off point were 0.575 (range, $0.498-0.651$ ) and $4.420 \times 10^{9} / \mathrm{L}$ for the change of WBC count, 0.725 (range, $0.660-0.790$ ) and $4.355 \mathrm{mmol} / \mathrm{L}$ for the change of blood glucose level, and 0.658 (range, 0.590-0.727) and $-11.900 \mathrm{mmol} / \mathrm{L}$ for the change of serum ALB level, respectively.

Patients with were assigned a score of 1 for each of the following factors: poor coughing ability, smoking habit, the change of WBC count and blood glucose levels greater than the cut-off values, and the change of ALB level lower than the 


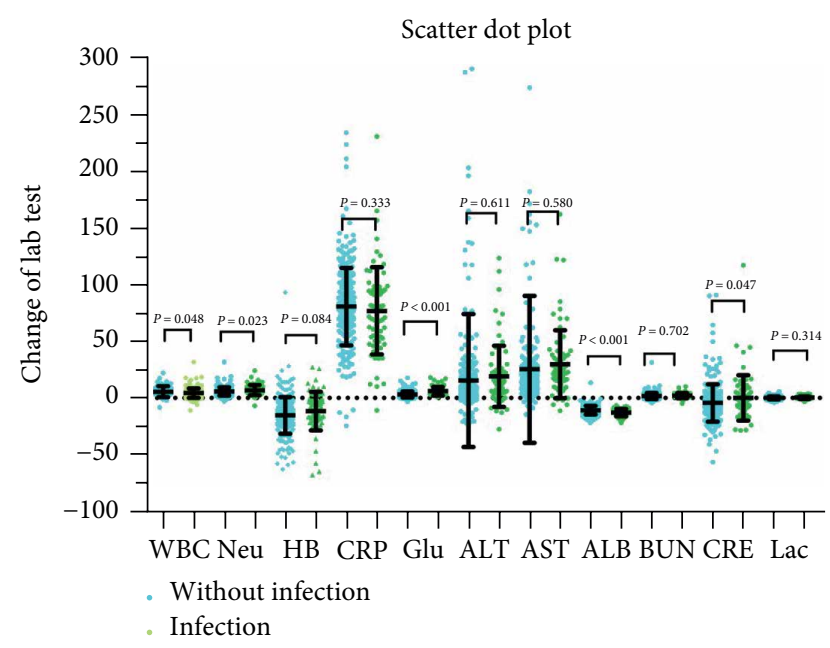

FIGURE 3: Scatter dot plot of the change of laboratory tests between two groups.

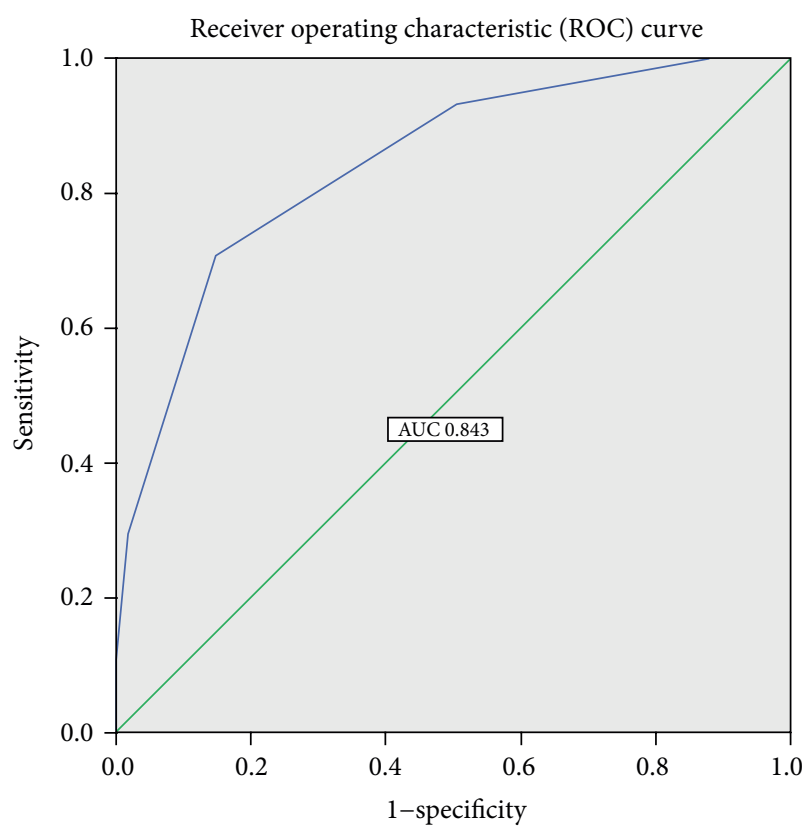

FIGURE 4: Receiver operating characteristic (ROC) curve of the scoring system.

cut-off value; patients who did not meet these requirements were assigned a score of 0 each.

The AUC of this predictive model was 0.843 (range, $0.793-$ 0.894 ); the sensitivity, specificity, and cut-off score were $70.8 \%$, $85.3 \%$, and 2.500, respectively (Figure 4 and Table 5).

3.6. Pulmonary Complication through Clinical Diagnosis. The differences of changes of laboratory tests in the groups divided by pulmonary complication were showed in the supplementary data.

\section{Discussion}

Results of comparing the infection and noninfection groups in the present study indicated that poor coughing ability, smoking status, the change of WBC count, the change of ALB level, and the change of blood glucose level were independent risk factors for predicting postoperative infection in patients undergoing McKeown esophagogastrectomy.

According to our analysis, smoking acts as one of the independent risk factors for predicting postoperative infection. Liu et al. [5] reported that smoking history was one of the risk factors of postoperative lung infection. Moreover, the study conducted by Kinugasa et al. [6] showed that smoking habit was risk factors for postoperative pulmonary complications. Furtermore, the similar result shown in the study conducted by Ferguson et al. [7]. Smoking history could increase airway resistance, then cause numerous postoperative sputum thrombi. Consequently, the risk of pulmonary infection increases by impairment of the respiratory epithelium cilia structure, damaging to goblet cells, and weakening cilia movement.

The result of our study showed that the change of WBC count was associated with the postoperative infection. Sugita et al. [8] showed that the preoperative WBC count did not differ between infected and noninfected patients, although the POD1 WBC count was significantly higher in infected patients than in noninfected patients. The study conducted by Gomez et al. [9] also showed that the median WBC count was significantly greater in patients with infection than in those without infection during the first 10 postoperative days.

Furthermore, we found that the change of ALB level was an independent risk factor for postoperative infection in patients underwent McKeown esophagogastrectomy. The study conducted by Yin et al. [10] showed that low serum albumin was independently associated with the surgical site infections (SSI). Moreover, Zhao et al. [11] demonstrated that ALB level $<35 \mathrm{~g} / \mathrm{L}$ was an independent risk factor for postoperative infectious complications in patients with hepatocellular cancer. In addition, Yuwen et al. [12] showed that an ALB level of $<35 \mathrm{~g} / \mathrm{L}$ was associated with an increased risk of SSI in patients after orthopedic operations.

Lastly, our study showed that the change of blood glucose level was an independent risk factor for predicting infection. Similar to our findings, Vriesendorp et al. [13] indicated that the POD1 blood glucose level in esophageal cancer patients after esophagectomy was only associated with the length of hospitalization. Moreover, $\mathrm{Ng}$ et al. [14] showed that the change in the target glucose control in diabetic patients was independently associated with an increase in SSI. Another study conducted by Ambiru et al. [15] demonstrated that the SSI rates were correlated with the hyperglycemia following surgery.

In the scoring system of our study, the continuous variables changed to categorical variables through the cut-off value (higher than cut-off value is number $A$, lower than the cut-off value is number $\mathrm{B}$ ), and added value for each factor. All the categorical variables used assigns $20 \%$ for each factor in the total score because it was useful and easy in clinical practice.

I think our study is quite novelty. On the one hand, our study talked about specific disease (esophageal cancer), and undergoing specific kind of operation (McKeown 
TABLE 2: Difference in perioperative features among patients who underwent McKeon esophagogastrectomy.

\begin{tabular}{|c|c|c|c|c|}
\hline Outcome & Infection group (\%) & Noninfection group (\%) & Statistic ( $\chi^{2}$ or $T$ value $)$ & $P$ value \\
\hline Total no. of patients & 72 & 286 & & \\
\hline Hoarseness & & & 12.282 & $<0.001^{*}$ \\
\hline Yes & $17(23.6)$ & $25(8.7)$ & & \\
\hline No & $55(76.4)$ & $261(91.3)$ & & \\
\hline Poor coughing ability & & & 70.967 & $<0.001^{*}$ \\
\hline Yes & $37(51.4)$ & $26(9.1)$ & & \\
\hline No & $35(48.6)$ & $260(90.9)$ & & \\
\hline Wound pain & & & 0.050 & 0.823 \\
\hline Yes & $14(19.4)$ & $59(20.6)$ & & \\
\hline No & $58(80.6)$ & $227(79.4)$ & & \\
\hline Chest pain/chest distress & & & 0.290 & 0.590 \\
\hline Yes & $1(1.4)$ & $2(0.7)$ & & \\
\hline No & $71(98.6)$ & $284(99.3)$ & & \\
\hline Heart rate & & & 3.586 & 0.058 \\
\hline$>100 /$ min & $16(22.2)$ & $7(2.4)$ & & \\
\hline$\leq 100 / \mathrm{min}$ & $56(77.8)$ & $279(97.6)$ & & \\
\hline Respiratory rate & & & 0.567 & 0.451 \\
\hline$>24 / \mathrm{min}$ & $3(4.2)$ & $7(2.4)$ & & \\
\hline$\leq 24 / \mathrm{min}$ & $69(95.8)$ & $279(97.6)$ & & \\
\hline Atrial fibrillation & & & 0.000 & 0.993 \\
\hline Yes & $2(2.8)$ & $8(2.8)$ & & \\
\hline No & $70(97.2)$ & $278(97.2)$ & & \\
\hline$M A P$ & $90.20 \pm 9.83$ & $89.83 \pm 10.33$ & -0.281 & 0.779 \\
\hline
\end{tabular}

*Statistically significant at $P \leq 0.05$. MAP: Mean artery pressure

TABle 3: Difference in the change laboratory test results between the infection and noninfection group (POD1 minus Pre-McKeown esophagogastrectomy).

\begin{tabular}{|c|c|c|c|c|}
\hline & Infection group & Noninfection group & $T$ value & $P$ value \\
\hline $\mathrm{WBC}\left(\times 10^{9} / \mathrm{L}\right)$ & $5.59 \pm 4.75$ & $4.47 \pm 4.14$ & -1.987 & $0.048^{*}$ \\
\hline Neutrophils $\left(\times 10^{9} / \mathrm{L}\right)$ & $7.00 \pm 4.63$ & $5.83 \pm 3.69$ & -2.276 & $0.023^{*}$ \\
\hline $\mathrm{HB}(\mathrm{g} / \mathrm{L})$ & $-11.55 \pm 17.01$ & $-15.29 \pm 16.21$ & -1.735 & 0.084 \\
\hline Serum ALB (g/L) & $-12.83 \pm 3.45$ & $-10.69 \pm 3.86$ & 4.295 & $<0.001^{*}$ \\
\hline $\operatorname{ALT}(\mathrm{IU} / \mathrm{L})$ & $19.18 \pm 26.88$ & $15.57 \pm 58.71$ & -0.509 & 0.611 \\
\hline AST (IU/L) & $29.72 \pm 29.96$ & $25.38 \pm 64.76$ & -0.554 & 0.580 \\
\hline BUN (mmol/L) & $2.06 \pm 2.28$ & $1.88 \pm 2.77$ & -0.514 & 0.702 \\
\hline $\mathrm{CRE}(\mu \mathrm{mol} / \mathrm{L})$ & $0.17 \pm 19.94$ & $-4.02 \pm 15.40$ & -1.996 & $0.047^{*}$ \\
\hline Glucose (mmol/L) & $6.03 \pm 3.97$ & $3.78 \pm 3.18$ & -4.525 & $<0.001^{*}$ \\
\hline $\mathrm{CRP}(\mathrm{mg} / \mathrm{L})$ & $76.97 \pm 38.48$ & $81.21 \pm 32.93$ & 0.968 & 0.333 \\
\hline Lactic acid (mmol/L) & $0.34 \pm 1.32$ & $0.18 \pm 1.21$ & -1.007 & 0.314 \\
\hline
\end{tabular}

*Statistically significant at $P \leq 0.05$. WBC, white blood cell; HB, hemoglobin; AST, aspartate aminotransferase; ALT, alanine aminotransferase; ALB, serum albumin; BUN, blood urea nitrogen; CRE, creatinine; CRP, C-reactive protein.

esophagogastrectomy); on the other hand, in our study, we used the changes of these laboratory tests different from other studies.

The limit of our study is that it is a single center retrospective research, and the study population comprised only Asian participants. The patient number enrolled in our study was relatively small, so some risk factor, such as alcohol consumption were not included in the independent risk factors. Probably, in the future study, we could get more data to conduct a tendentious matching analysis to better identify the risk factors. Moreover, we do not have postoperative pulmonary function tests in our clinical practice; we need prospective study to address this problem for better diagnosing the respiratory complication, which is one of the major complications of esophagectomy $[16,17]$. Our study did not compare the minimally invasive esophagectomy with open invasive esophagectomy, some studies showed that minimally invasive esophagectomy had lower incidence of postoperative infection than open invasive esophagectomy [18-20]. In addition, other factors should be contained in further study, such as operation time, intraoperative bleeding, the application of antacids, and so on. 
TABLE 4: Multivariate logistic regression analysis of the risk factors for infections after McKeown esophagogastrectomy.

\begin{tabular}{|c|c|c|c|c|c|c|}
\hline \multirow{2}{*}{ Variate } & \multicolumn{3}{|c|}{ Univariate analysis } & \multicolumn{3}{|c|}{ Multivariate analysis } \\
\hline & $P$ & OR & $95 \% \mathrm{CI}$ & $P$ & OR & $95 \% \mathrm{CI}$ \\
\hline Gender & 0.016 & 0.415 & $(0.203-0.850)$ & & & \\
\hline Smoking habit & $<0.001^{*}$ & 2.727 & $(1.584-4.695)$ & $<0.001^{*}$ & 4.218 & $(2.110-8.431)$ \\
\hline Poor coughing ability & $<0.001^{*}$ & 10.571 & $(5.725-19.520)$ & $<0.001^{*}$ & 11.034 & $(5.358-22.724)$ \\
\hline Hoarseness & $<0.001^{*}$ & 3.227 & $(1.633-6.378)$ & & & \\
\hline Change of ALB level & $<0.001^{*}$ & 0.855 & $(0.795-0.921)$ & $=0.001^{*}$ & 0.849 & $(0.772-0.935)$ \\
\hline Change of WBC & 0.051 & 1.059 & $(1.000-1.123)$ & $=0.050$ & 1.079 & $(1.000-1.164)$ \\
\hline Change of neu & 0.027 & 1.073 & $(1.008-1.143)$ & & & \\
\hline Change of CRE & 0.059 & 1.013 & $(1.000-1.027)$ & & & \\
\hline Change of glucose & $<0.001^{*}$ & 1.283 & $(1.177-1.398)$ & $<0.001^{*}$ & 1.237 & $(1.117-1.371)$ \\
\hline
\end{tabular}

*Statistically significant at $P \leq 0.05$. Change of ALB: serum ALB level within $24 \mathrm{~h}$ after surgery minus pre-operation. Change of WBC level: serum WBC level within $24 \mathrm{~h}$ after surgery minus pre-operation. Change of neutropils: serum neutropils level within $24 \mathrm{~h}$ after surgery minus pre-operation. Change of CRE: serum CRE level within $24 \mathrm{~h}$ after surgery minus pre-operation. Change of glucose: blood glucose level within $24 \mathrm{~h}$ after surgery minus pre-operation. Factors were entered into multivariate regression using the forward stepwise (conditional) approach $(P \leq 0.05)$.

TABLE 5: Receiver operating characteristics of the independent risk factors and the scoring system.

\begin{tabular}{|c|c|c|c|c|c|}
\hline Factors & AUC (95\% CI) & $P$ value & Cut-off & Sensitivity (\%) & Specificity (\%) \\
\hline Change of WBC level & $0.575(0.498-0.651)$ & $0.039^{*}$ & 4.420 & 61.1 & 53.8 \\
\hline Change of glucose level & $0.725(0.660-0.790)$ & $<0.001^{*}$ & 4.355 & 62.5 & 74.8 \\
\hline Change of ALB level & $0.658(0.590-0.727)$ & $<0.001^{*}$ & -11.900 & 59.7 & 67.1 \\
\hline Scoring system & $0.843(0.793-0.894)$ & $<0.001^{*}$ & 2.5 & $70.8 \%$ & $85.3 \%$ \\
\hline
\end{tabular}

*Statistically significant at $P \leq 0.05$. Change of ALB: serum ALB level within $24 \mathrm{~h}$ after surgery minus pre-operation. Change of WBC level: serum WBC level within $24 \mathrm{~h}$ after surgery minus pre-operation. Change of glucose: blood glucose level within $24 \mathrm{~h}$ after surgery minus pre-operation. With regard to the scoring system, patients were assigned a score of 1 for each of the following factors: poor coughing ability, smoking habit, change of WBC count and blood glucose levels greater than the cut-off values, and change of ALB level lower than the cut-off value, whereas patients who did not meet these requirements were assigned a score of 0 each.

\section{Conclusion}

Patients are exposed to high risks of predicting postoperative infection after McKeown esophagogastrectomy, although poor coughing ability, smoking habit, the change of WBC count, the change of ALB level, and the change of blood glucose level may be as independent risk factors for postoperative infections in these patients. At last, we used a scoring system comprising these 5 factors, and observed that the AUC of this predictive model was 0.843 (range, $0.793-0.894$ ), whereas the sensitivity, specificity, and cut-off score were $70.8 \%, 85.3 \%$, and 2.500 , respectively.

\section{Data Availability}

The datasets used and/or analyzed in the current study are available from the corresponding author upon request.

\section{Consent}

All authors have agreed to the publication of this manuscript.

\section{Conflicts of Interest}

The authors declare that they have no conflicts of interest.

\section{Authors' Contributions}

Chongxiang Chen designed the study. Chongxiang Chen, Qingyu Zhao designed the search strategy and performed the search. Chongxiang Chen, and Tianmeng Wen performed abstract screening, full text screening, data extraction, and risk of bias assessment. Chongxiang Chen and Qingyu Zhao drafted the manuscript. All authors revised the manuscript, as well as reading and approving the final manuscript.

\section{Acknowledgments}

We acknowledge all the contributed authors.

\section{Supplementary Materials}

The supplementary data includes a table of the difference of the changes of laboratory tests divided by clinical diagnosis pulmonary complication. (Supplementery Materials)

\section{References}

[1] A. Jemal, F. Bray, M. M. Center, J. Ferlay, E. Ward, and D. Forman, "Global cancer statistics," CA: A Cancer Journal for Clinicans, vol. 61, no. 2, pp. 69-90, 2011. 
[2] W. Chen, R. Zheng, S. Zhang et al., "Report of incidence and mortality in China cancer registries, 2009," Chinese Journal of Cancer Research, vol. 25, no. 1, pp. 10-21, 2013.

[3] R. C. Connors, B. C. Reuben, L. A. Neumayer, and D. A. Bull, "Comparing outcomes after transthoracic and transhiatal esophagectomy: a 5-year prospective cohort of 17,395 patients," Journal of the American College of Surgeons, vol. 205, no. 6, pp. 735-740, 2007.

[4] C. D. Wright, J. C. Kucharczuk, S. M. O'Brien, J. D. Grab, and M. S. Allen, "Predictors of major morbidity and mortality after esophagectomy for esophageal cancer: a society of thoracic surgeons general thoracic surgery database risk adjustment model," The Journal of Thoracic and Cardiovascular Surgery, vol. 137, no. 3, pp. 587-595, 2009, discussion 596.

[5] G. Liu, L. Peng, B. Liu, K. Wang, and Y. Han, "Analysis of risk factors for pulmonary infection in patients with minimally invasive esophagectomy," Oncology Letters, vol. 17, no. 3, pp. 3283-3288, 2019.

[6] S. Kinugasa, M. Tachibana, H. Yoshimura et al., "Postoperative pulmonary complications are associated with worse short- and long-term outcomes after extended esophagectomy," Journal of Surgical Oncology, vol. 88, no. 2, pp. 71-77, 2004.

[7] M. K. Ferguson, A. D. Celauro, and V. Prachand, "Prediction of major pulmonary complications after esophagectomy," The Annals of Thoracic Surgery, vol. 91, no. 5, pp. 1494-1500, 2011, discussion 1500-1501.

[8] S. Sugita, T. Hozumi, K. Yamakawa, T. Goto, and T. Kondo, "White blood cell count and C-reactive protein variations following posterior surgery with intraoperative radiotherapy for spinal metastasis," Clinical Spine Surgery, vol. 30, no. 8, pp. E1022-E102, 2017.

[9] E. Gomez, M. Heredia, P. Jorge et al., "Use of procalcitonin and white blood cells as combined predictors of infection in cardiac surgery patients," Critical Care, vol. 18, no. Suppl 1, p. P215, 2014.

[10] L. X. Yin, B. M. Chen, G. F. Zhao, Q. F. Yuan, Q. Xue, and $\mathrm{K} . \mathrm{Xu}$, "Scoring system to predict the risk of surgical site infection in patients with esophageal cancer after esophagectomy with cervical anastomosis," Surgical Infections, vol. 19, no. 7, pp. 696-703, 2018.

[11] Y. Zhao, Y. Jin, and Y. Wu, "Postoperative infectious complications after liver resection for hepatocellular carcinoma," Journal of Cancer Research and Therapeutics, vol. 12, no. 8, pp. C268-C270, 2016.

[12] P. Yuwen, W. Chen, H. Lv et al., "Albumin and surgical site infection risk in orthopaedics: a meta-analysis," BMC Surgery, vol. 17, no. 1, Article ID 7, 2017.

[13] T. M. Vriesendorp, J. H. DeVries, J. B. Hulscher, F. Holleman, J. J. van Lanschot, and J. B. Hoekstra, "Early postoperative hyperglycaemia is not a risk factor for infectious complications and prolonged in-hospital stay in patients undergoing oesophagectomy: a retrospective analysis of a prospective trial," Critical Care, vol. 8, no. 6, pp. R437-442, 2004.

[14] R. R. G. Ng, A. Myat Oo, W. Liu, T. E. Tan, L. K. Ti, and S. T. H. Chew, "Changing glucose control target and risk of surgical site infection in a Southeast Asian population," The Journal of Thoracic and Cardiovascular Surgery, vol. 149, no. 1, pp. 323-328, 2015.

[15] S. Ambiru, A. Kato, and F. Kimura et al., "Poor postoperative blood glucose control increases surgical site infections after surgery for hepato-biliary-pancreatic cancer: a prospective study in a high-volume institute in Japan," Journal of Hospital Infection, vol. 68, no. 3, pp. 230-233, 2008.

[16] C. E. Avendano, P. A. Flume, G. A. Silvestri, L. B. King, and C. E. Reed, "Pulmonary complications after esophagectomy," The Annals of Thoracic Surgery, vol. 73, no. 3, pp. 922-926, 2002.

[17] P. Dumont, J. Wihlm, J. Hentz, N. Roeslin, R. Lion, and G. Morand, "Respiratory complications after surgical treatment of esophageal cancer. A study of 309 patients according to the type of resection," European Journal of Cardio-Thoracic Surgery: Official Journal of the European Association for Cardio-Thoracic Surgery, vol. 9, no. 10, pp. 539-543, 1995.

[18] Z. Zhang, M. Xu, M. Guo, and X. Liu, "Long-term outcomes of minimally invasive Ivor Lewis esophagostomy for esophageal squamous cell carcinoma: compared with open approach," International Journal of Surgery, vol. 45, pp. 98-104, 2017.

[19] J. Wang and M. Q. Xu, M. R. Xie, X. Y. Mei, "Minimally invasive ivor-lewis esophagectomy (MIILE): a single-center experience," The Indian Journal of Surgery, vol. 79, no. 4, pp. 319-325, 2017.

[20] S. S. Biere, M. I. van Berge Henegouwen, L. Bonavina, C. Rosman et al., "Predictive factors for postoperative respiratory infections after esophagectomy for esophageal cancer: outcome of randomized trial," Journal of Thoracic Disease, vol. 9, no. Suppl 8, pp. S861-S867, 2017. 


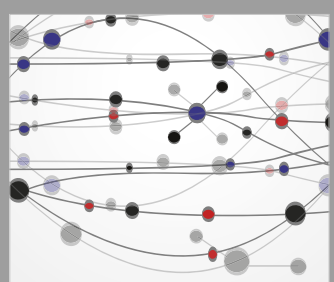

The Scientific World Journal
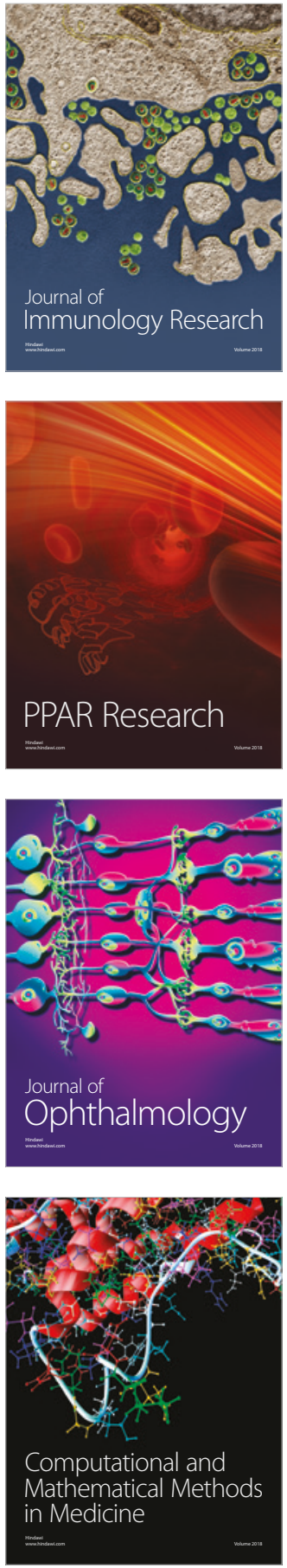

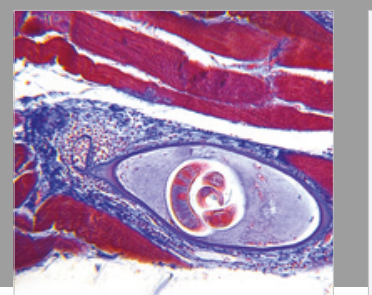

Gastroenterology Research and Practice

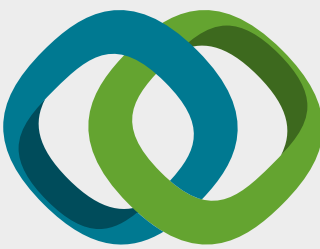

\section{Hindawi}

Submit your manuscripts at

www.hindawi.com
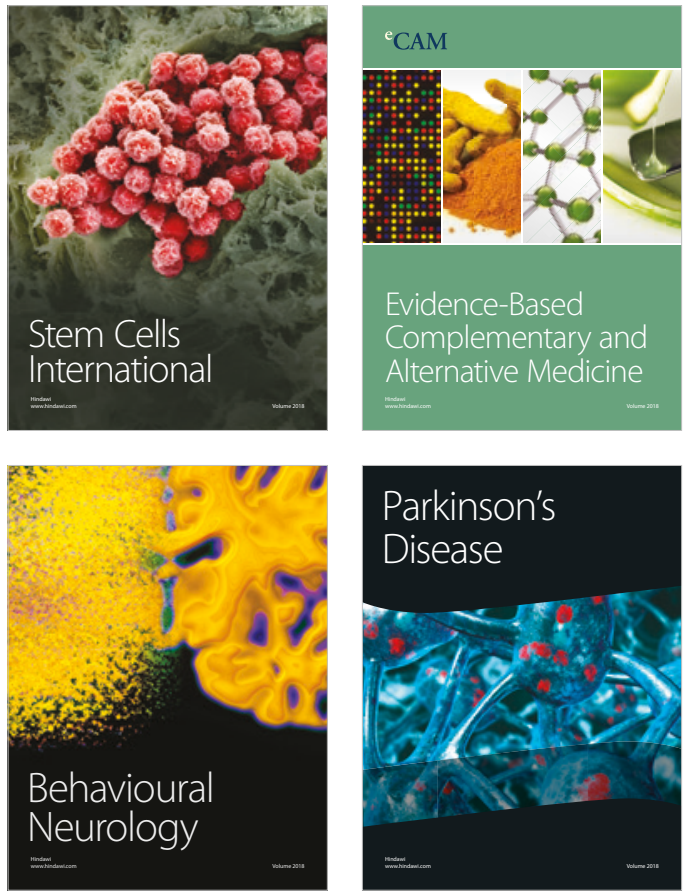

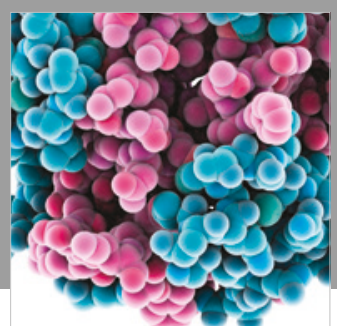

ournal of

Diabetes Research

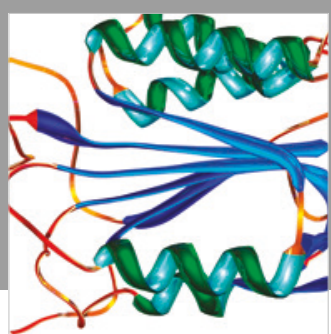

Disease Markers
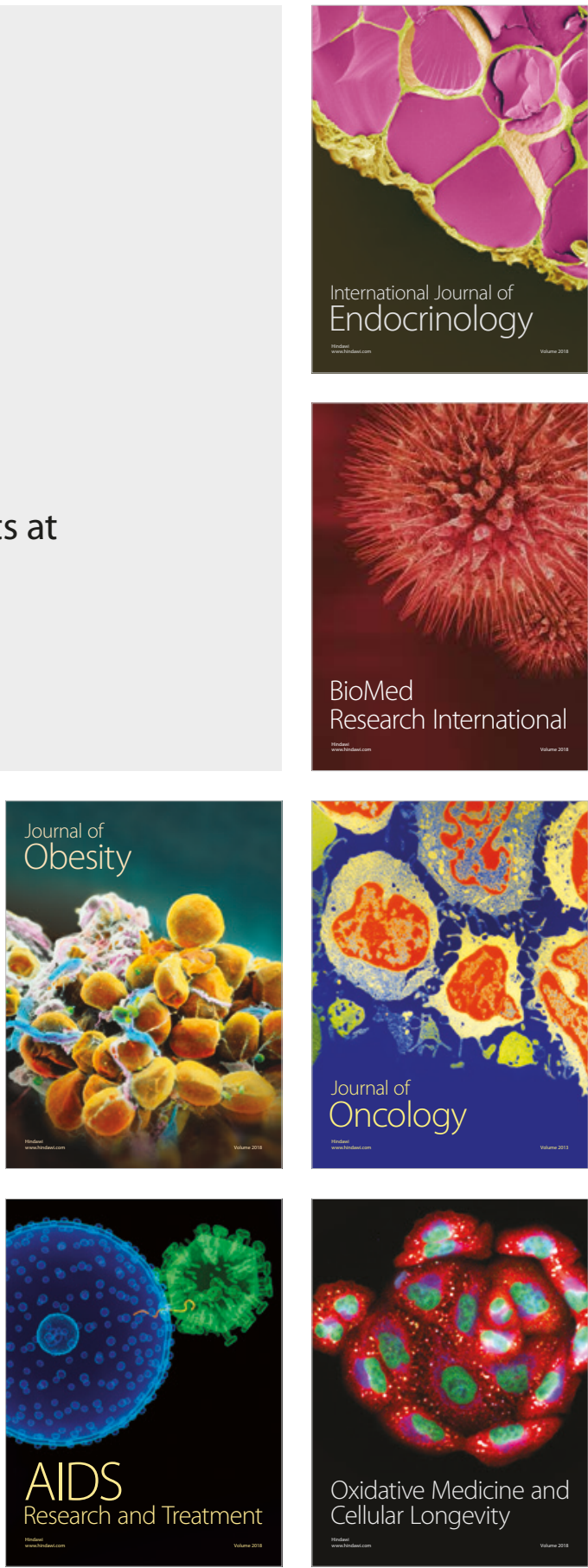